\title{
One-stage reconstruction of neck burns with single- layer dermal matrix
}

\author{
Jorge Luis Gaviria1, Viviana Gómez-Ortega ${ }^{2}$ \\ 'Department of Plastic and Reconstructive Surgery, Burn Unit Hospital Simón Bolivar, Bogotá 110131, Colombia. \\ 2Department of Plastic and Reconstructive Surgery, Fundación Santa Fé de Bogotá, Bogotá 110111, Colombia.
}

Correspondence to: Prof. Jorge Luis Gaviria, Department of Plastic and Reconstructive Surgery, Burn Unit of the Health Services Unit of Simón Bolívar North Subnetwork E.S.E, Bogotá 110131, Colombia. E-mail: gaviriajorgeluis@yahoo.com

How to cite this article: Gaviria JL, Gómez-Ortega V. One-stage reconstruction of neck burns with single-layer dermal matrix. Plast Aesthet Res 2018;5:35. http://dx.doi.org/10.20517/2347-9264.2018.38

Received: 24 May 2018 First Decision: 24 Jul 2018 Revised: 30 Aug 2018 Accepted: 1 Sep 2018 Published: 27 Sep 2018

Science Editor: Raúl González-García Copy Editor: Yuan-Li Wang Production Editor: Huan-Liang Wu

\begin{abstract}
Aim: The aim of the study was to describe the applicability of a single-layer acellular dermal matrix, in combination with split-thickness skin graft (STSG) in one-stage surgical procedure in patients with deep neck second - and third - degree burns.
\end{abstract}

Methods: A descriptive longitudinal study was conducted at the Burn Unit of the Health Services Unit of Simón Bolívar North Subnetwork E.S.E. of the Secretariat of Health in Bogotá, Colombia, from January 12016 to December 312017 in which we describe the applicability of a single-layer acellular dermal matrix in combination with STSG in one-stage surgical procedure in patients with deep neck second and third degree burns.

Results: A total of 9 patients were treated. Exposed areas required definitive coverage using a single-layer dermal regeneration matrix and autografts in a one-stage procedure, following excision of keloid scars and scar retractions ( $n$ $=7$ ), and in the case of acute-phase deep burn wounds, following complete necrotic tissue removal $(n=2)$. No patient presented infection during postoperative follow-up. At 2 months postoperatively, stability of treated area and adequate resistance of the skin substitute and autografts could be observed. They appeared normal in color, with no degree of contractures or functional limitations.

Conclusion: This is the first study demonstrating that the use of a single-layer acellular dermal matrix template for one stage reconstruction of post-burn full thickness neck defects is an effective, safe and excellent reconstructive option. The use of an artificial dermal matrix in one-step surgical approach, allows rapid healing and early mobilization of the neck, and in selective cases, it may reduce the need for local-regional or free flap coverage; moreover, it is associated with excellent skin formation, good functional and esthetic results and minimal donor site morbidity. 
Keywords: Neck, burns, matrix, template, reconstruction

\section{INTRODUCTION}

Modern burn care is based on operative wound management. There is clear evidence that immediate excision and closure are lifesaving for patients.

Significant reconstructive and rehabilitative challenges associated with neck burns must be addressed in aesthetic units. Acute care will greatly influence the succeeding scarring, reconstructive needs, and longterm outcomes. In most cases, reconstruction will encompass the restoration of both form and function of soft tissue. The procedures used will highly depend on the level of local scarring ${ }^{[1]}$.

The neck is a difficult area to reconstruct because of its cylinder-like dynamic structure. Severe neck burns are followed by various expected deformities: neck contracture with limited range of motion, problems with oral ability, and chin contracture with eversion of the lower lip, affecting both eating and speech. Thus, the initial reconstruction method selected by the surgeon will determine the functional and final aesthetic result ${ }^{[2]}$.

Neck reconstruction techniques are conditional on the extent of the release and type of defect, as well as on the institution's financial resources. In small areas having no exposed deep structures, full-thickness skin grafts are a good option, whereas for larger defects with no exposed deep structures, split-thickness skin grafts (STSGs) are indicated. Regional skin expansion is also described, along with free flaps, including the anterolateral thigh flap and the thoracodorsal artery perforator flap in the case of exposed deep structures. Normally, reconstruction following neck contracture release with STSGs produces poor results due to an elevated rate of recontractures. Greenwood and Mackie ${ }^{[3]}$ attribute this phenomenon to natural graft contractile tendency, to pain precluding mobility, and to platysma contraction. Better results have been reported with full-thickness skin grafts, with the drawback of limited donor sites, particularly in young and lean patients.

Skin substitutes are another alternative for neck reconstruction. They have the advantage of being simpler procedures, compared to free flaps, exhibiting less secondary contracture than STSGs, and offering usability in large neck reconstruction areas ${ }^{[4]}$.

The implementation of dermal templates to effectively treat wounds is based on an appropriate debridement and a well-vascularized host bed. Applying dermal templates on contaminated wounds entails a high risk of infection, since dermal templates have a limited ability to fight infection. Once it has been applied, fibroblasts, endothelial cells, and inflammatory cells migrate into and repopulate the dermal template, ultimately replacing the scaffold. A thin STSG may be applied for wound coverage after template take in large wounds. The dermal template may be grafted at the time of its application in a well-vascularized wound if a thin scaffold is used ${ }^{[5]}$.

There are only a few published studies that have used a dermal matrix with a skin autograft in a one-stage repair. In 2011, Greenwood and Mackie ${ }^{[3]}$ reported 1 case, in which Matriderm collagen/elastin dermal matrix plus STSGs were used in a one-stage repair after a neck contracture release, yielding positive results. Other substitutes, such as Glyaderm, based on glycerinized donor skin, Integra ${ }^{\circledR}$ bilayer, and biodegradable polyurethane dermal substitute, have been used for neck reconstruction. However, all of them require two surgical procedures, where a skin graft is placed 3 weeks following take of dermal matrix ${ }^{[6-8]}$.

Another study published by Seo et al. ${ }^{[9]}$ in 2014 describes a retrospective analysis of 28 patients with postburn severe cervical contractures, which were reconstructed in a single-stage procedure with skin substi- 
tutes and STSGs, yielding an overall take rate of $95.9 \%$, with only 1 patient showing recontracture. Nevertheless, all patients from this study underwent late reconstraction, considering that the contracture was already present as a burn sequela. To date there are no reports in the international literature demonstrating this type of management in patients with acute burns, achieving positive results. In this regard, the present study reports such management not only in 7 patients with irregular scarring but also in 2 patients exhibiting acute burns.

The aim of this prospective, single-center, observational study was to describe the application of a singlelayer acellular dermal matrix, in combination with STSGs, in a one-stage surgical procedure in patients with deep partial- and full-thickness neck burns, and sequelae.

\section{METHODS}

We report the use of a single-layer acellular dermal matrix (Integra ${ }^{\circ}$ ) for one-stage reconstruction of neck defects following a full-thickness burn.

A descriptive longitudinal study was conducted at the Burn Unit of the Health Services Unit of Simón Bolívar North Subnetwork E.S.E. of the Secretariat of Health in Bogotá, Colombia, from January 12016 to December 31 2017. The study was approved by the Institution's Ethics Committee, and it was based on the ethical principles contained in the Declaration of Helsinki.

The study included 9 patients with deep partial- or full-thickness burns of any etiology and extension, involving the neck either partially or totally, as well as patients who presented abnormal scarring on the neck with or without retraction, who required definitive coverage with skin grafts after complete excision of the scar lesion.

Patients were requested to sign an informed consent form to be able to participate in the study and have their pictures taken. Patients showing additional comorbidities, including malnutrition, high blood pressure, diabetes mellitus, and coagulation disorders, were excluded. Patients having severe mental disorders or exposed areas not suitable for graft coverage were also excluded.

\section{Surgical procedure}

The dermabrasion or complete eschar removal was performed under general anesthesia in deep partial- and full-thickness burns. After obtaining a clean wound free of necrotic tissue, the host area was rinsed with isotonic saline solution, and electrocautery was used for hemostasis. Once the burn area was prepared and ready, the single-layer acellular dermal matrix template was applied to the wound bed to achieve a complete substitute of the full-thickness dermis. The single-layer acellular dermal matrix template (Integra ${ }^{\circ}$ ) was placed, allowing $1 \mathrm{~cm}$ to extend beyond the wound edges. Next, the template was checked to ensure there were no bubbles or hematomas. Immediately after this step, a STSG of $0.010^{\prime \prime}$ was obtained from the dorsolateral thigh with the use of an electric dermatome and fixed in place with surgical staples. Specialized dressings were applied to the grafts in order to stabilize them and close the dead space, thereby lowering complication rates.

Wound dressings were first uncovered on the 5th day and changed every 5 days until complete take of the dermal matrix and skin autograft. Blisters, small hematomas or seromas were drained during dressing change. In case of partial graft loss, the site was regrafted or closure was done by applying cultured autologous keratinocytes, thus, avoiding healing by secondary intention. Monitoring was conducted at 1, 2, 3, 6, 9, and 12 months, and evolution was recorded through photography. 
An admission assessment was performed for each patient, and based on these findings, a physical and occupational therapy plan was designed with the aim of decreasing pain and edema, improving ranges of motion, and controlling positioning and functionality for neck rotation and extension. Both the physical and occupational therapies were suspended during the first 5 postoperative days and resumed with physical reconditioning activities and orthotic management with splints in extension $\left(110^{\circ}\right)$, acupressure on the scar, and the use of Lycra garments, silicone, lubricants, and moisturizers. Additionally, hypertrophic, red, inflexible, firm, and raised scars were treated with steroid injections.

\section{Statistical analysis}

The socio-demographic variables age and sex were registered. Other clinical characteristics were registered as well, including total burned body surface area using the Lund and Browder chart, burn depth, size of the defect following complete necrotic tissue removal or total scar excision, amount of dermal matrix used in square centimetres, autograft take percentage, and complications. Scars were assessed at month 1 and month 12. For qualitative variables, frequencies and percentages were used, whereas for quantitative variables, mean and range were preferred. Microsoft $\mathrm{Excel}^{\circledR}$ was used.

\section{RESULTS}

A total of 9 patients were treated. Exposed areas required definitive coverage using a single-layer dermal regeneration matrix and autografts in a one-stage procedure, following excision of keloid scars and scar retractions $(n=7)$, and in the case of acute-phase deep burn wounds, following complete necrotic tissue removal $(n=2)$. In this group, 5 patients were 1 woman and 4 men, with a mean age of 29.6 years (range 13-48 years).

Mean total body surface area affected was 30\% (range 8\%-70\%). Most patients showed additional burns involving the face, anterior chest and upper extremities, exhibiting deep partial- and full-thickness burns.

Mean treated area was $144 \mathrm{~cm}^{2}$. No patient presented infection during postoperative follow-up. At 2 months postoperatively, stability of treated area and adequate resistance of the skin substitute and autografts could be observed. They appeared normal in color, with no degree of contractures or functional limitations. The graft was smooth, flat, non-indurated, and elastic, and its color was very similar to the surrounding area. Such characteristics improved over time [Figures 1 and 2].

Four of the nine patients showed signs of hypertrophic scarring at the edges of the graft, particularly in the junction between the dermal matrix and normal skin, consisting of hyperemic, raised, and slightly indurated scars having a width of less than $5 \mathrm{~mm}$. They responded adequately to management with steroid injections and the use of Lycra garments, silicone gel, and acupressure.

One patient presented autograft loss of $5 \mathrm{~cm}^{2}$ at immediate postoperative follow-up, which was successfully managed by applying cultured autologous keratinocytes [Figure 3].

\section{DISCUSSION}

Neck burns are difficult to reconstruct because of the cylinder-like structure and constant movement of the neck. Among the most common challenges associated with deep neck burns is the high frequency of contractures and functional impairments. Such deformities cause prolonged disabilities and social rejection. Although multiple procedures have been described, including Z-plasty, W-plasty, local flaps, and free flaps, STSGs continue to be recognized as the standard treatment, particularly for deep and extensive burns. Skin substitutes have improved the quality of STSGs significantly, reducing and preventing recontractures ${ }^{[5]}$. Additionally, they are a good alternative for this area. 

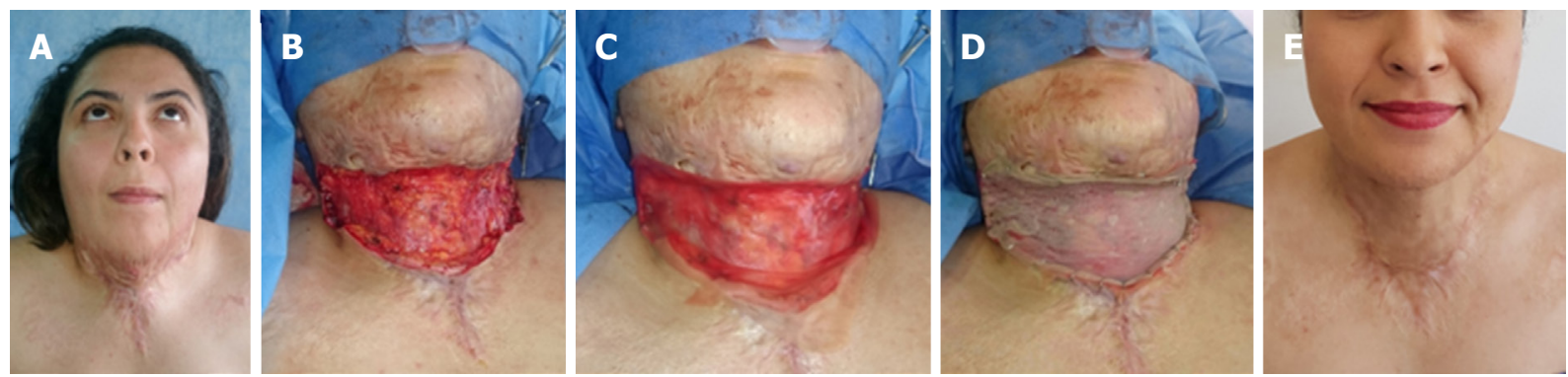

Figure 1. A: A 25-year-old woman, who suffered thermal burn caused by alcohol, with a deep partial- and full-thickness burn covering $30 \%$ TBSA. There were injuries to the face, neck, anterior chest, and upper limbs. She presented severe mentocervical contracture; B: the photograph shows the area following excision of the scar; C: the single-layer dermal matrix is shown in place; D: the split-thickness skin graft may be observed over the template; E: outcome at 12 months postoperatively after reconstruction with $135 \mathrm{~cm}^{2}$ of acellular dermal matrix and autografts in a single-step procedure, without functional limitations. TBSA: total body surface area
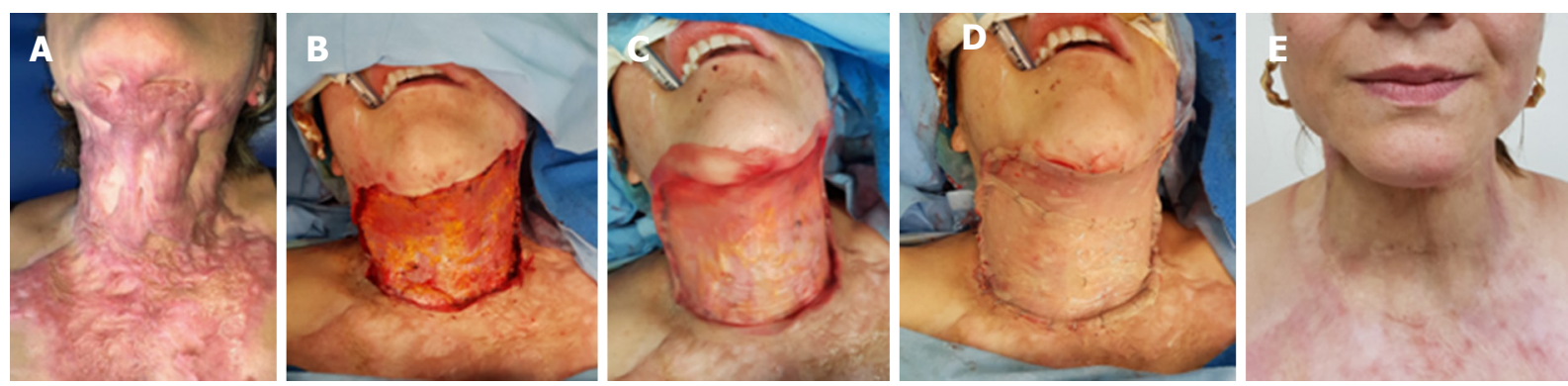

Figure 2. A: A 38-year-old woman, who suffered thermal burn caused by mineral spirits, with full-thickness burns that developed in multiple keloid scars in the mentocervical region; B: defect after excision of keloid scars; C: the photograph shows the application of the single-layer dermal matrix over the defect; D: result after placing $206 \mathrm{~cm}^{2}$ of dermal regeneration matrix and autografts in a single-step procedure; E: Appearance at 10 months postoperatively. She did not present any functional limitations
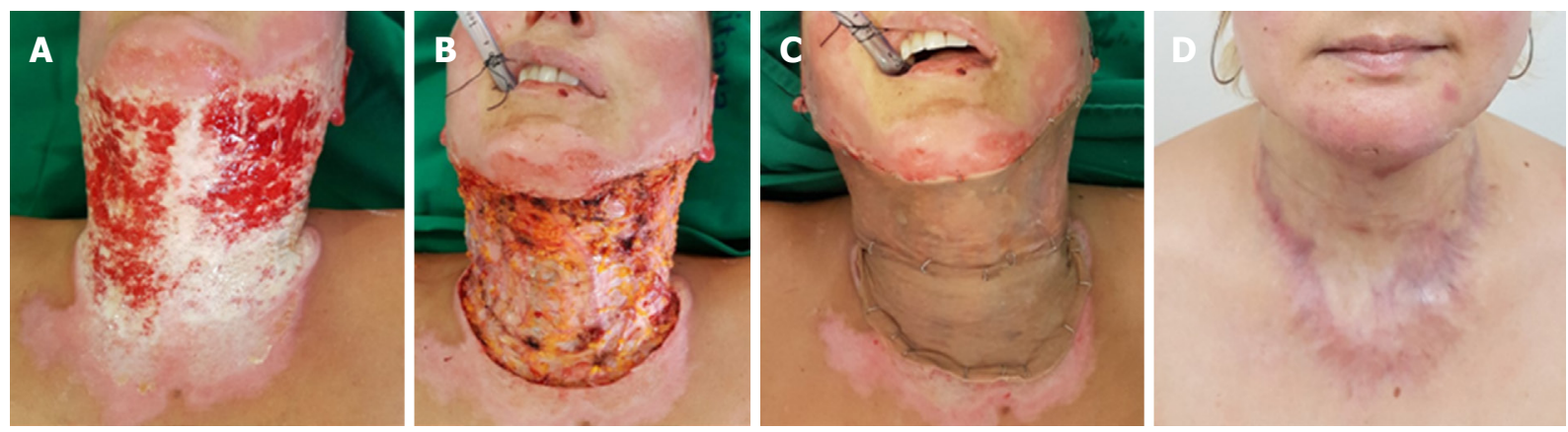

Figure 3. A: A 38-year-old woman, who suffered thermal burn caused by alcohol, with a full-thickness burn covering 30\% TBSA; B: injured area after eschar removal; C: reconstruction with $210 \mathrm{~cm}^{2}$ of dermal matrix and autografts. She presented $5-\mathrm{cm}^{2}$ graft loss that was treated with autologous keratinocyte cultures; D: the picture shows the appearance at 9 months postoperatively. TBSA: total body surface area

Recently, there has been an increased number of reports describing the use of several skin substitutes as a good reconstruction alternative. Some of them, such as Glyaderm, Integra bilayer, and biodegradable polyurethane dermal substitute, require a two-stage procedure consisting initially of a dermal matrix implantation and subsequently of skin grafting once there is complete take of the matrix. Seo et al ${ }^{[9]}$ show a report of 24 neck reconstructions with a $95.9 \%$ take rate, under the use of AlloDerm and Matriderm, by placing the skin graft immediately after the dermal matrix implantation in a single-stage procedure. 
Even though this study is a preliminary report of a short series of 9 cases, the results obtained allow the development of comparative studies encompassing the different techniques used for post-burn neck reconstructions, comparing them with STSGs or other skin substitutes. It is worth noting that this is the first case series demonstrating that the use of a single-layer acellular dermal matrix (Integra ${ }^{\circ}$ ) in a one-stage reconstruction of neck defects following a full-thickness burn is an effective, safe and excellent reconstructive option in managing both acute burns and sequelae.

Along the same lines of the results described by Seo et al. ${ }^{[9]}$ with AlloDerm and Matriderm, the use of Integra Single Layer in this study yielded comparable results, including the following: short operating room times, with no complications and excellent functional and cosmetic results in such a difficult-to-treat area. As may be observed from the photographs presented, there is good color match in the grafted area. The use of skin substitutes offers alternative solutions aimed at successfully treating complex soft-tissue defects, optimizing the quality of the reconstructed $\operatorname{skin}^{[10-12]}$.

In Colombia, the other skin substitute available is the Integra bilaminar dermal regeneration matrix within the framework of current practice regarding skin substitutes, consisting of a two-stage surgical procedure: application of the dermal regeneration template to the wound and, following a 2-4 weeks' interval, the replacement of the silicone pseudoepidermal layer of the template with a thin-thickness skin autograft. The increased number of surgeries, a prolonged hospitalization and the need of additional immobilization are patent disadvantages of this two-step repair. Furthermore, the time required for the dermal matrix to fully integrate causes prolongation of the inflammatory phase, increasing the possibility of fibrosis and scar re$\operatorname{traction}^{[13-15]}$. The Integra ${ }^{\otimes}$ bilaminar dermal matrix has proven to entail a higher risk of infection, as a 3-week interval is required to allow take before placing skin autografts. Lohana et al ${ }^{[16]}$ report that the risk of infection is higher in such cases.

In the case of deep and extensive neck burns, where insufficient donor skin is available for full-thickness skin grafts, which require coverage with STSGs, we consider that the use of dermal matrices may be contemplated as a first surgical option to improve STSG outcomes. This would decrease the high postoperative contracture rate and poor functional results observed in these areas.

A parameter that we consider to be important for the success of this procedure is the preparation of a wellvascularized, non-infected wound bed, before applying the dermal matrix and skin graft. Adequate surgical debridement is crucial and mandatory. Stabilizing the single-layer acellular dermal matrix and the skin graft through the use of pressure dressings is also essential for a successful outcome.

No unstable or keloid scars were recorded; pliable skin and excellent elasticity of grafted areas was documented, without scar contractures or serious functional limitations. Cosmetic appearance of grafted sites was equally acceptable.

In conclusion, our efforts were centered on choosing the appropriate techniques that will provide good functional and cosmetic results. The use of an artificial dermal matrix in a one-step surgical approach allows rapid healing and early mobilization of the neck, and in some cases, it may reduce the need for local or regional flap or free flap coverage. Moreover, it is associated with excellent skin formation, good functional and aesthetic results, and low donor-site morbidity. Therefore, it should be considered as an alternative to other reconstruction techniques, particularly when covering highly demanding areas, such as the neck.

\section{DECLARATIONS}

\section{Authors' contributions}

Concept and design, data analysis, manuscript preparation, critical revision and finalizing of the manuscript: Gaviria JL, Gómez-Ortega V

Data acquisition: Gaviria JL 


\section{Availability of data and materials}

We confirm that the data were strictly obtained from medical records according to the privacy policy and ethics code of our institute.

\section{Financial support and sponsorship}

None.

\section{Conflicts of interest}

All authors declared that there are no conflicts of interest.

\section{Ethical approval and consent to participate}

The study was approved by the Institution's Ethics Committee. Ethical approval number UI-18-15. All participants were adults, and they all signed an informed consent form before participating in the study.

\section{Consent for publication}

Patients were requested to sign an informed consent form to be able to participate in the study and have their pictures taken.

\section{Copyright}

(c) The Author(s) 2018.

\section{REFERENCES}

1. Jones I, Currie L, Martin R. A guide to biological skin substitutes. Br J Plast Surg 2002;55:185-93.

2. Shores JT, Gabriel A, Gupta S. Skin substitutes and alternatives: a review. Adv Skin Wound Care 2007;20:493-508.

3. Greenwood JE, Mackie IP. Neck contracture release with matriderm collagen/elastin dermal matrix. Eplasty 2011;11:e16.

4. Böttcher-Haberzeth S, Biedermann T, Schiestl C, Hartmann-Fritsch F, Schneider J, Reichmann E, Meuli M. Matriderm ${ }^{\circledR} 1 \mathrm{~mm}$ versus integra ${ }^{\circledR}$ single layer $1.3 \mathrm{~mm}$ for one-step closure of full thickness skin defects: a comparative experimental study in rats. Pediatr Surg Int 2012;28:171-7.

5. Burke JF, Yannas IV, Quinby WC Jr, Bondoc CC, Jung WK. Successful use of a physiologically acceptable artificial skin in the treatment of extensive burn injury. Ann Surg 1981;194:413-28.

6. Wagstaff MJ, Caplash Y, Greenwood JE. Reconstruction of an anterior cervical necrotizing fasciitis defect using a biodegradable polyurethane dermal substitute. Eplasty 2017;17:e3.

7. Hunt JA, Moisidis E, Haertsch P. Initial experience of Integra in the treatment of post-burn anterior cervical neck contracture. Br J Plast Surg 2000;53:652-8.

8. Pirayesh A, Richters CD, Hoeksema H, Verbelen J, Heyneman A, Monstrey S. Clinical evaluation of glyaderm, a dermal subsitute based on glycerinized donor skin. Available from: https://www.intechopen.com/books/skin-grafts-indications-applications-and-currentresearch/clinical-evaluation-of-glyaderm-a-dermal-substitute-based-on-glycerinized-donor-skin. [Last accessed on 16 Sep 2018]

9. Seo DK, Kym D, Hur J. Management of neck contractures by single-stage dermal substitutes and skin grafting in extensive burn patients. Ann Surg Treat Res 2014;87:253-9.

10. Moiemen NS, Vlachou E, Staiano JJ, Thawy Y, Frame JD. Reconstructive surgery with integra dermal regeneration template: histologic study, clinical evaluation, and current practice. Plast Reconstr Surg 2006;117:160S-74S.

11. Heimbach D, Luterman A, Burke J, Cram A, Herndon D, Hunt J, Jordan M, McManus W, Solem L, Warden G. Artificial dermis for major burns. A multi-center randomized clinical trial. Ann Surg 1998;208:313-20.

12. Stern R, McPherson M, Longaker MT. Histologic study of artificial skin used in the treatment of full-thickness thermal injury. J Burn Care Rehabil 1990;11:7-13.

13. Heimbach DM, Warden GD, Luterman A, Jordan MH, Ozobia N, Ryan CM, Voigt DW, Hickerson WL, Saffle JR, DeClement FA, Sheridan RL, Dimick AR. Multicenter postapproval clinical trial of Integra dermal regeneration template for burn treatment. J Burn Care Rehabil 2003;24:42-8.

14. Yannas IV, Burke JF, Orgill DP, Skrabut EM. Wound tissue can utilize a polymeric template to synthesize a functional extension of skin. Science 1982;215:174-6.

15. Yannas IV, Lee E, Orgill DP, Skrabut EM, Murphy GF. Synthesis and characterization of a model extracellular matrix that induces partial regeneration of adult mammalian skin. Proc Natl Acad Sci U S A 1989;86:933-7.

16. Lohana P, Hassan S, Watson SB. IntegraTM in burns reconstruction: our experience and report of an unusual immunological reaction. Ann Burns Fire Disasters 2014;27:17-21. 